Paper presented at PED 2010, NIST, Maryland USA, March 8-10 2010

\title{
Collection of Evacuation Data for Large Passenger Vessels at Sea
}

\author{
E.R. Galea ${ }^{1}$, R.C. Brown ${ }^{1,2}$, L. Filippidis ${ }^{1}$, and S. Deere ${ }^{1}$ \\ 1 Fire Safety Engineering Group, University of Greenwich, London, UK \\ 2 Offshore Safety and Survival Centre, Fisheries and Marine Institute, Memo- \\ rial University, St. John's, Canada \\ Corresponding author: E.R.Galea@gre.ac.uk
}

\begin{abstract}
In the past decade, significant effort has gone into the planning and execution of full-scale sea trials in an attempt to improve, calibrate and validate existing evacuation models for passenger ships. In September, 2009 two assembly exercises were conducted at sea onboard the RO-PAX ferry SuperSpeed 1 by team members of the EU-funded project SAFEGUARD. The exercises were conducted with passengers during routine sailings between the ports of Kristiansand, Norway and Hirtshals, Denmark. Between both trials, a total of 1,769 passengers were assembled, on day one, 902 passengers and on day two 867 passengers. As part of the data collection exercise, passenger response time data was collected - using video cameras - and passenger movement data was collected using a novel infrared (IR) based position logging system. This paper briefly describes the development and testing of the data acquisition system and briefly discusses preliminary results.
\end{abstract}

\section{Introduction}

Understanding how people behave in emergency situations within maritime settings is vital if we are to; design and develop evacuation efficient vessels and evacuation procedures, train crew in the management of evacuation situations, develop reliable ship evacuation models and regulate the design and operation of vessels. An essential component of this understanding is the collection and characterisation of human performance data. Unfortunately, little data relating to passenger response time or full-scale validation data in maritime environments exists. In the first International Maritime Organisation (IMO) document to specify protocols for the use of ship evacuation models in the analysis and certification of passenger ship design, IMO MSC Circ. 1033 [1], an arbitrary uniform random distribution was set to represent the response time behaviour of passengers. It has been shown that this is unrepresentative of actual passenger response time and liable to produce incorrect or misleading conclusions concerning the suitability of ship design for evacuation [2]. As part of the EU Framework V project FIRE EXIT [3], passenger response time data was collected for a passenger ship at sea [3,4]. This 
data was accepted by the IMO and used in the formulation of IMO MSC Circ. 1238 [5], the modified protocols for passenger ship evacuation analysis and certification. However, the response time data produced by FIRE EXIT $[3,4]$ related to only a single passenger vessel. As such the data cannot be considered representative of passenger ships in general. The IMO Fire Protection (FP) SubCommittee in their modification of MSC Circ. 1033 at the FP51 meeting in February 2007 [6] invited member governments to provide, “...further information on additional scenarios for evacuation analysis and full scale data to be used for validation and calibration purposes of the draft revised interim guideline". To this end, project SAFEGUARD was proposed and successfully funded through the EU framework 7 programme. The project aims to address this IMO requirement by providing relevant full-scale data and proposing and investigating additional benchmark scenarios that can be used in certification analysis. Six full-scale data sets will be collected as part of SAFEGUARD - two trials on each of three different types of passenger vessels.

This paper concentrates on the first two data sets collected on the first vessel - a large RO-PAX ferry operated by Color Line AS called SuperSpeed 1. The vessel can carry approximately 2000 passengers and crew and over 700 vehicles. It operates on the route between Kristiansand in Norway and Hirtshals in Denmark, a trip of 3 hours and 15 minutes. The ship contains a mixture of spaces spread over three decks including; business and traveller class seating areas (airline style seating), large retail and restaurant/cafeteria areas, bar areas, indoor and outdoor general seating areas and general circulation spaces. The ship has four assembly stations, three located on Deck 7 (assembly stations A, B and C) and one located on Deck 8 (assembly station D). Assembly stations B and C are located on the outer decks while assembly stations $\mathrm{A}$ and $\mathrm{D}$ are internal.

Three types of data sets were collected in each trial. The first consisted of response time data collected using video cameras positioned throughout the vessel. Some 30 battery powered mini digital video cameras were used to collect the response time data (see Figure 1). The cameras were placed at strategic locations throughout the vessel to record not only the time at which the passengers respond, but also the nature of the activities that they were involved in at the time. The second type of data collected comprised validation data for ship based evacuation models. This consisted of starting locations of passengers, arrival time at the designated assembly locations and the paths taken by the passengers from the start location to the assembly location. This data was collected using a novel data acquisition system consisting of 30 Infra-Red (IR) beacons, each emitting unique IR signals and data logging tags worn by each passenger (see Figure 2). The third type of data consisted of a questionnaire completed by each of the participants. 

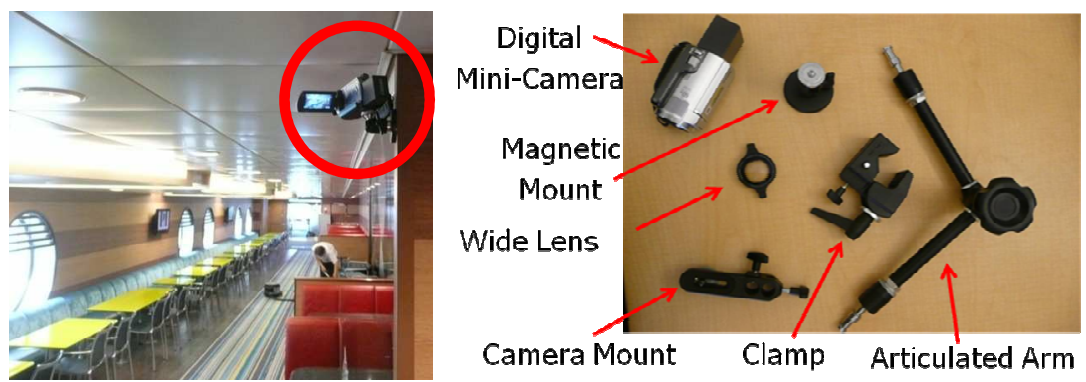

Fig. 1. Example video camera mounting location (left, circled) and mounting equipment with camera (right).
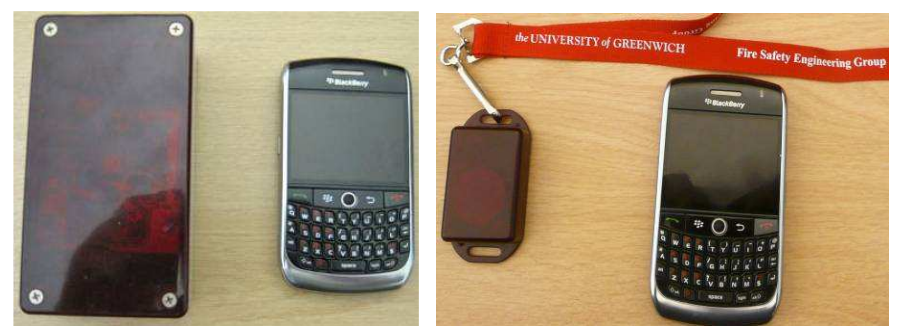

Fig. 2. IR field generating beacon (left) and IR logging tag (right).

\section{Data Collection Methodology}

Here we describe the process of collecting the validation data. Previous efforts at collecting comprehensive full-scale ship evacuation validation data have been less than successful due to the complication of the associated data analysis. Previous efforts have attempted to use video footage to manually track individuals through the vessel [3]. However, tracking individuals through the complex layout of a large passenger vessel is extremely time consuming. Depending on the complexity of the structure, the analyst may have to track an individual through tens of different video camera locations. Attempting to track a handful of individuals this way can be extremely tedious and prone to error. In the case of SuperSpeed, tracking almost 1000 passengers across three large decks would have been unthinkable! Automated video tracking systems also have problems as they require a 'birds eye' view of the targeted individuals if they are to accurately monitor an individual's progress through a particular location, making installation of the video equipment difficult due to the low head room often found on ships [7]. Furthermore, the problem still persists of tracking individuals passing through many different camera locations.

A comprehensive investigation was undertaken of technologies which may be useful in addressing this problem. This identified two specific technologies - passive 
radio frequency identification (RFID) and infra-red (IR) position logging. Both systems rely on similar underlying concepts - devices are mounted throughout the structure that generate uniquely identified radio frequency or IR fields or "gates" and passengers wear a device that allows for their unique identification as they move throughout the structure and pass through each gate. In this way, if the structure is instrumented with a sufficient number of gates, then as a person moves around the structure, their tag either logs or permits the logging of the ID for the gates that were passed and at what time (see Figure 3).

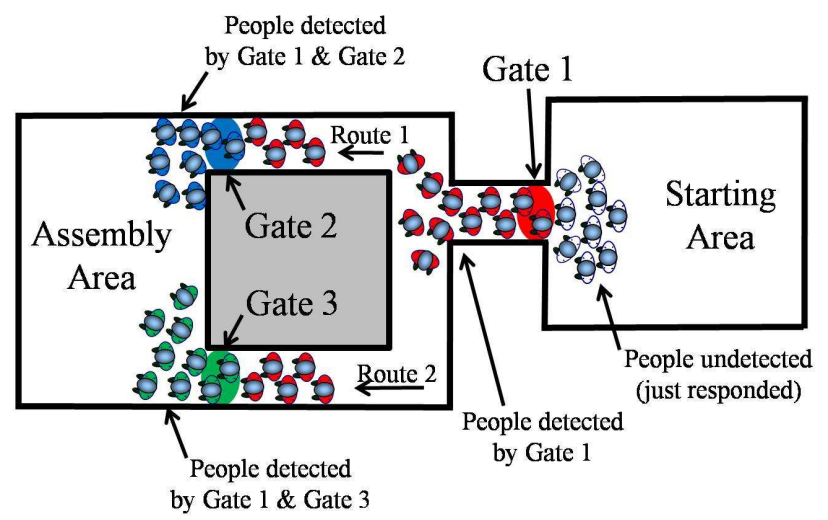

Fig. 3. Example of tracking system following individuals from a starting area to an assembly area or exit point via two different routes.

Both systems rely on the population agreeing to wear a tag for the purposes of the trial. As the trial may take place at any time, the participant population must be prepared to wear the tag for an extended period of time (possibly all day/night) and so it must not interfere with normal operations, be comfortable, and if possible must blend in with their normal attire. For example, attaching the tags to a hat or cap, while ideal for detection, would not be acceptable.

There are fundamental differences between the RFID and IR systems, particularly the way in which the gates are generated and the way in which communication takes place between the tag and gate for logging of position and time. The passive RFID technology examined (see Figure 4) relies on a pair of antennae that generate a radio frequency (RF) field (gate) with sufficient power to energise tags that enter it. The tags use the RF energy from the field to wirelessly transmit a signal to the receiving antennae (the same antennae that generate the RF field) which then sends the information to a processor that logs the tag's unique ID and the time at which the signal was received. For this system, the RFID tag acts as a passive device with no built-in power source and no data storage capacity. The data for people tracked is stored either by the component that generates the RF field or an attached computer. The main difficulty with this technology is that human bodies attenuate RF signals, sometimes in an unpredictable manner, thus making the placement of RFID antennae and tags of critical importance. In large crowds, es- 
pecially, data can be lost creating inaccuracies in the validation dataset. In a recent series of evacuation trials using RFID [8,9], it is suggested that read rates "will be better than $50 \%$ if proper alignment and measurement power of reader antennas is found through experiments" in crowded situations [8]. The success rate is also critically dependent on how the tag is worn, whether it is in contact with the skin, near metal objects or concealed by clothing. In addition, RFID systems tend to be bulky for temporary applications and logistics of setup becomes time consuming due to the need to carefully run cables and ensure suitable antennae orientations. Further, while RFID tags tend to be inexpensive in large quantities required for test series, they can be damaged relatively easily jeopardising the quality of the dataset. Considerable effort is required to select the correct tag and form factor to ensure the highest read-rate possible.
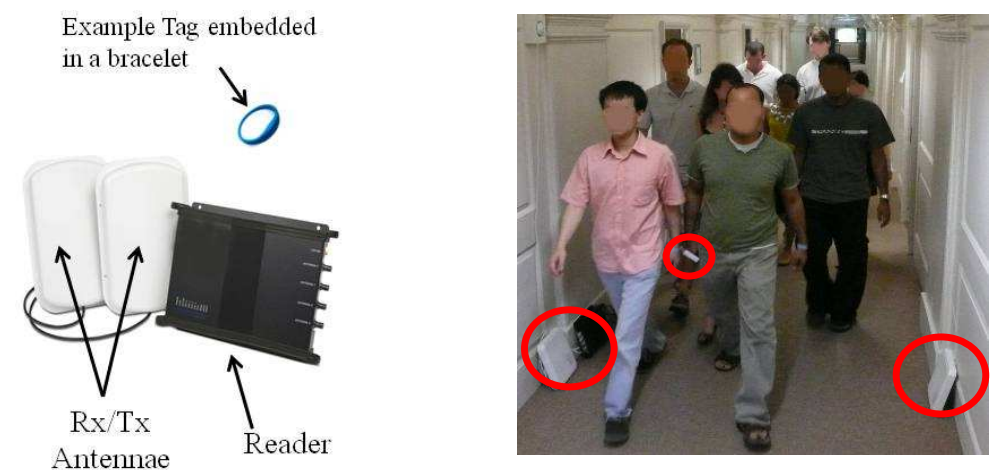

Fig. 4. Example RFID system tested (left) and example of field test (right).

A series of tests was undertaken of a representative RFID system, first in a corridor at the University of Greenwich and then on-board the SuperSpeed vessel. The RFID system was manufactured by Alien Technology Corp. and consisted of an Alien model ALR- 8800 reader and a pair of Alien model ALR-8610 circular polarised multistatic antennae (i.e. capable of both generating the RF field and receiving tag transmission data for logging). This system was designed to operate in the European UHF band in the $865.7-867.5 \mathrm{MHz}$ range with power levels of $2 \mathrm{~W}$ ERP and is compliant with European radio regulations. The system was designed to read EPC Class 1 Generation 2 UHF tags. In addition to the RFID system, various types of EPC Class1, Gen2 UHF tags were purchased in different form factors, specifically; peel and stick labels, silicone rubber wristbands and plastic wrist/ankle hospital-style bands. The corridor tests consisted of some 12 people walking down a $1.89 \mathrm{~m}$ wide corridor of which all 12 were wearing the RFID tags. Trials with both wrist and ankle bands were undertaken. Subjects were asked to walk together past the antennae as a group keeping their speed, position and group density consistent from test to test (see Figure 4). Tag reads were stored on a computer for each test. The maximum read rate was $75 \%$ for tests where subjects were permitted to walk normally (i.e. with arms swinging by their side). One test 
case was conducted where the subjects were asked to fold their arms, thereby shielding the tags somewhat. For this test, it was found that read rate decreased significantly to $17 \%$. A series of trials was also conducted on-board the SuperSpeed. Unfortunately, the trials on-board SuperSpeed were conducted using only nine people (equal to the number of tags) in an open space and so do not represent a reliable test under crowded situations, nevertheless, these trials returned an average read rate of $86 \%$. The ship trials demonstrated that the RFID system could work within the confines of the metal environment of a passenger ship. The corridor tests suggest that read rates of up to $75 \%$ can be achieved using the RFID technology in crowded situations.

The IR technology examined (see Figure 2) relies on a beacon that generates an IR light field (gate). As a tagged individual passes through the field, IR light sensors in the tag detect the IR light and log its ID and the time at which it was detected in the tag's own internal memory. For the IR system, no data is transmitted from the tag. Following the test, tags must be retrieved in order to determine the occupant's route data. The main disadvantage with this technology is that occupant route data is not collected unless tags are returned following the test. In addition, the IR tag is also considerably more expensive than the passive RFID tag, costing approximately 15 times more. This is very different than the (passive) RFID tags which, due to their low individual price and inability to log route data, do not need to be returned at the end of the tests. However, the IR tags, if collected after the trial, can be reused time and time again. In addition, the disadvantage of the IR tag turns into an advantage for the IR gate beacons. As the path history of the individual is recorded on the tag itself, this simplifies the nature of the IR beacons, reducing their cost (by a factor of approximately 60), as well as their size and power requirements compared to the RFID system. This allows many IR beacons to be placed through the structure, thus allowing for more granular definition of occupant routes. In addition, the beacons are much easier to set-up, greatly simplifying instrumentation setup and due to their reduced size, simplifying logistics involved in transporting the equipment to the test site.

The IR system was manufactured by RFID Centre Ltd. A modified version of their TagMobile system was employed which includes IR generating beacons and logging tags which are hung around the neck using a lanyard. The RFID Centre worked with FSEG to modify this system to make it more appropriate for use in evacuation applications. This involved a redesign of the standard IR tag. The modified IR system was put through a similar series of tests to the RFID system. In corridor tests at the University of Greenwich, the beacon was mounted on one side of the corridor at a height of $2.13 \mathrm{~m}$ above the floor, facing perpendicular to the opposite wall. A total of ten tags were used and a group of 23 individuals was formed with the ten tagged subjects mixed throughout. The group was instructed to walk past the beacon keeping speed, position and group density consistent from test to test (see Figure 5). Subjects were asked to raise their hand when the tag indicated a successful read. These tests always returned a $100 \%$ read rate. Ship 
board tests using the IR system involved 10 test subjects wearing the IR tags. These tests returned an average of $93.5 \%$ successful read rate however, as with the RFID trials on the ship, these trials were not under heavily crowded situations and so were not considered representative of the intended application. However, the tests demonstrated that the system could work in a ship board environment.

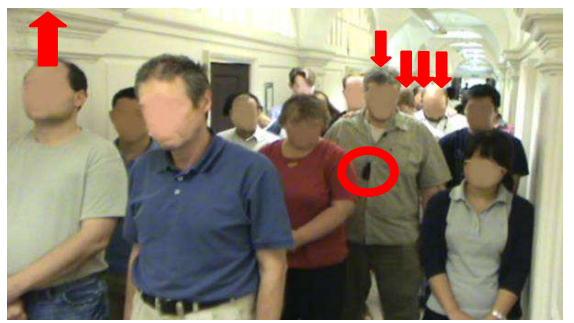

(a)

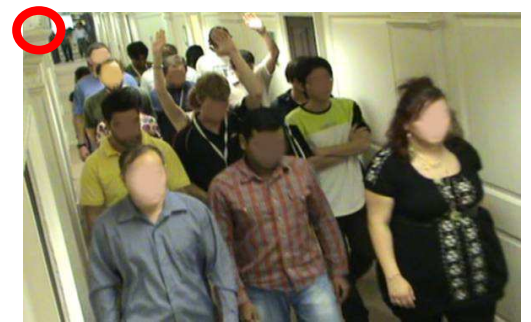

(b)

Fig. 5. IR corridor test (a) tagged people (down arrows), IR beacon out of view (up arrow) and IR tag (circled) (b) tagged people raising hands when tag is detected with IR beacon out of view (circled).

Based on the results generated in the various trials, it was concluded that the IRbased tracking system was better able to accurately track large numbers of individuals in high density crowds. While the RFID system provided reasonable read rates, performance of the IR system was superior in all cases. The success of the IR system is due in part to it not relying on direct line-of-sight between the beacon and the detector, the IR beams being readily reflected from surfaces and not distorted by human bodies. Furthermore, considering the logistical issues associated with using the two systems, the IR system was considered more versatile being; easier for setup and knock-down, not requiring external power supplies or cables, being relatively cheap to add additional gates and easier to transport. On completion of the trial, data is transferred from each retrieved tag to a computer via an IR reader. Software developed by FSEG then reads the tag information and, for each tag, identifies when the participant passed each numbered beacon and, for this application, when they arrived at the assembly station. Travel times, average travel speeds and levels of congestion can then be determined and associated with each beacon and hence beacon location. The data transfer process and path extraction is quick and reliable.

\section{Results and Discussion}

Both full-scale sea trials were conducted on the SuperSpeed's Kristiansand to Hirtshals crossing. While the passengers were aware that they would be involved in an assembly trial, they did not know at what time on the 3 hours and 15 minute crossing the trial would take place. Participation in the trials was not compulsory 
and children under the age of 12 were not permitted to take part. Passengers were given the IR tags as they boarded, together with an information sheet and asked to wear the IR tag at all times while on the vessel. The trials consisted of the ship's Captain sounding the alarm and crew moving the passengers into the designated assembly areas. The assembly trials were successfully conducted on the $4^{\text {th }}$ at 08:20 and $5^{\text {th }}$ at 08:19 of September 2009. On day 1 (day 2) there were 1431 (1349) passengers on board of which 1296 (1243) were eligible to participate in the trial. In total some 1170 (1192) IR tags were issued (some passengers refused to participate) of which 902 (867) passengers with IR tags participated in the trial in some capacity. Of these, a number of passengers who had been issued IR tags changed their minds and decided not to participate and so handed back their tags. Others with tags simply refused to participate while others responded, but then did not immediately move off to the assembly stations and so were not counted in the assembly total. The 902 (867) participating passengers represents $70 \%(70 \%)$ of the passengers on board and $77 \%$ (73\%) of the issued tags. In total $13(0.5 \%)$ of the tags were lost together with 60 lanyards. The first assembly trial was completed in $12 \mathrm{~min}$ while the second assembly trial was completed in $10 \mathrm{~min}$. The end of each assembly trial was determined by the Captain.

Here we consider some data extracted from the second trial. In 10 min some 841 passengers were assembled, this included 345 passengers who were already in the assembly stations and 496 passengers who made their way to the assembly stations. Depicted in Figure 6 is the assembly curve determined from the data collected by the IR tags. It displays the characteristic shape of a typical arrival curve. Note the passengers who assembled after $10 \mathrm{~min}$ (26 passengers) were not considered to be participating in the trial. A similar curve is available for each unique assembly station.

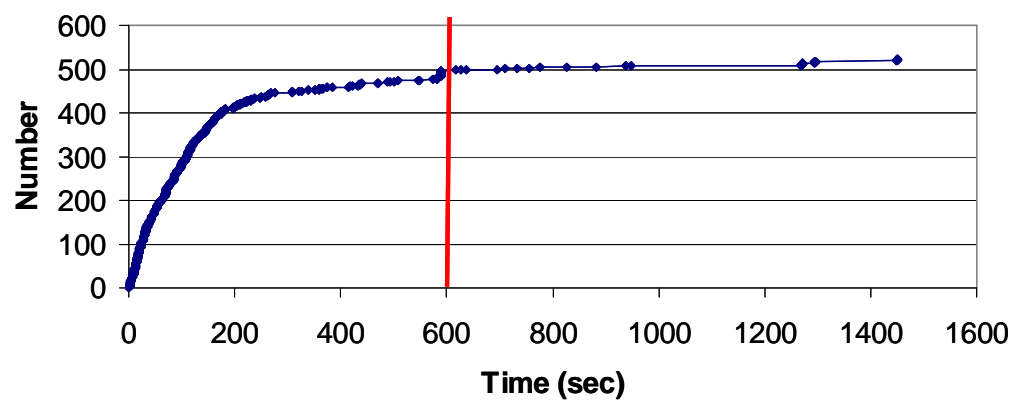

Fig. 6. Total assembly curve for trial 2.

Presented in Figure 7 is a typical path reconstructed from the tag information for a passenger. The passenger was located in the restaurant area and exited the restaurant, passing beacon 1469 secs after the sounding of the alarm, exiting the entrance to the dining area, passing beacon 1289 secs after the alarm and entering Assembly area D (end point), passing beacon 11103 secs after the sounding of the 
alarm. This type of analysis will enable an accurate data set to be assembled for model validation purposes, providing details of the routes taken by individual passengers, which assembly stations were used and associated assembly times. The collected data can also be used to generate approximate average travel speeds and in some cases population densities.

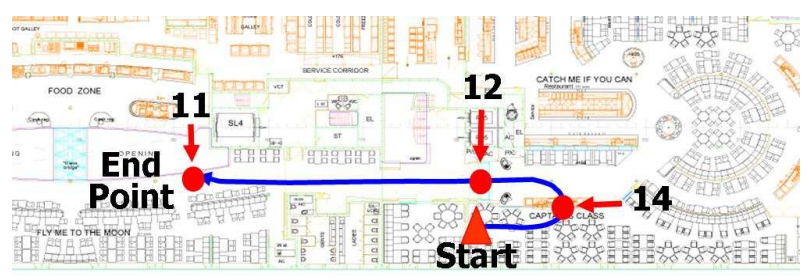

Fig. 7. Path adopted by a passenger during assembly trial 2

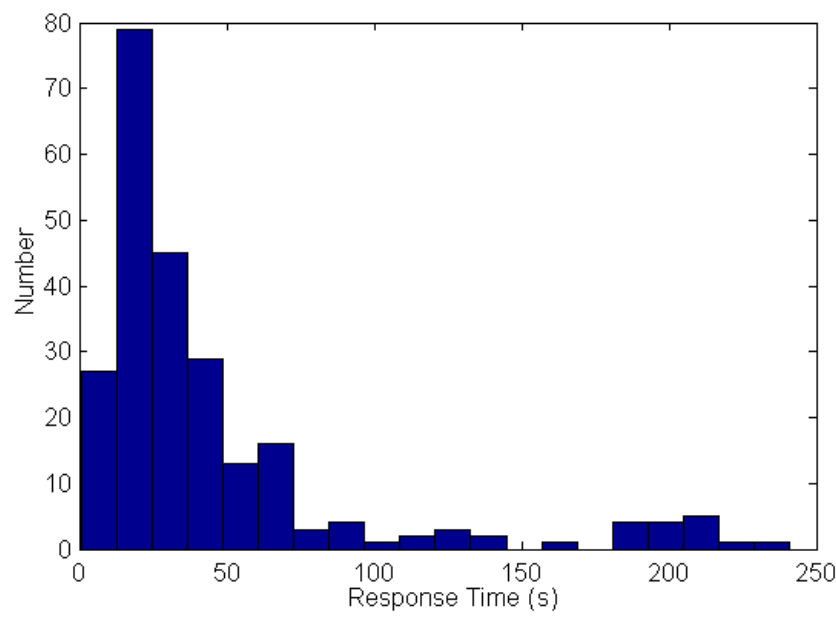

Fig. 8. Response time distribution derived from both trials for population in the bar region.

The travel speeds are only approximate as the size of a gate region, which is tuned as required for the specific location, can be between $4 \mathrm{~m}$ and $11 \mathrm{~m}$ wide. The population densities are determined using information concerning the number of people entering and leaving each gate region over a period of time. The accuracy of this measurement is improved if the flow is uni-directional and if densities are not extreme. The population response times are being determined by analysis of the video footage. This analysis is not only determining the response times but also the nature of the activities that the population was engaged in during the response phase, including determining the number of information and action tasks completed by each individual during the response phase. Shown in Figure 8 is the 
response time distribution for people located in the bar area. It displays the typical log-normal shape with a mean of 45.2 secs and a standard deviation of 48.6 secs.

\section{Conclusions}

Two assembly trials have been successfully conducted at sea involving a total of 1769 passengers. The data collected from these trials is being used to formulate a validation data set for ship evacuation models and to produce a more representative response time distribution for ship applications. As part of this project a new technology to track individuals through a complex structure, consisting of IR tags and beacons, was developed and tested. The technology is able to reliably track large numbers of people through complex structures, is relatively easy to setup and enables the rapid extraction of individual person trajectories.

\section{References}

1. IMO, Interim Guidelines for Evacuation Analyses for New and Existing Passenger Ships, IMO MSC/Circ 1033, 6 June 2002.

2. Deere, S., Galea, E.R., Lawrence, P., Filippidis, L. and Gwynne, S.: The impact of the passenger response time distribution on ship evacuation performance, Int J of Maritime Eng, Vol 148, Part A1, pp 35-44, (2006).

3. http://fseg.gre.ac.uk/fire/fire_exit_project.html

4. Galea, E.R., Deere, S., Sharp, G., Filippidis, L., Lawrence, P., and Gwynne, S.: Recommendations on the nature of the passenger response time distribution to be used in the MSC 1033 assembly time analysis based on data derived from sea trials.” Int J of Maritime Eng, Vol 149, Part A1, pp 15-29, (2007).

5. IMO MSC Circ 1238, 2007

6. FP 51/WP.3, 8 Feb 2007

7. Boltes, M., Seyfried, A., Steffen, B., and Schadschneider, A.: Automatic Extraction of Pedestrian Trajectories from Video Recordings. Proc Pedestrian and Evacuation Dynamics 2008, Springer, pp43-54, (2010).

8. Hostikka, S., Paloposki, T., Rinne, T., Saari, J.M. Korhonen, T. and Heliövaara, S.: Experimental Observations of Evacuation Situation. Pub. VTT Technical Research Centre of Finland, September, (2007).

9. Rinne, T., Hostikka, S., Paloposki, T., Korhonen, T., Saari, J.M. and Heliövaara, S.: Application of RFID and Video Imaging on Evacuation Observations in Offices and Public Buildings. Proceedings, $9^{\text {th }}$ IAFSS Symp, ED: B. Karlsson, pp 329-340, DOI:10.3801/IAFSS.FSS.9-329, (2008). 